Política y Estrategia

\title{
Evolución del comercio electrónico: una perspectiva desde China y América Latina
}

Camila Andrea Paipa Bolaños

Fundación Universitaria Empresarial de la Cámara de Comercio de Bogotá

\begin{abstract}
RESUMEN
El presente artículo analiza los efectos que ha tenido la apertura del comercio electrónico transfronterizo o e-commerce (electronic commerce) y las nuevas formas de generar relaciones comerciales internacionales tomando en cuenta el modelo económico de China, basado en exportaciones, el cual ha influido en el desarrollo de las economías emergentes de Latinoamérica, entre ellas, Brasil, México, Argentina, Colombia, Perú y Chile, lo que convierte a la región en una de las de mayor crecimiento en la nueva economía digital, gracias al flujo de mercados que, a su vez, permite generar nuevas estrategias de desarrollo tanto para el gigante asiático como para los países suramericanos mencionados.
\end{abstract}

\section{PALABRAS CLAVE}

China; comercio electrónico; desarrollo económico; estrategias de desarrollo; Latinoamérica.

\section{CITACIÓN}

Bolaños Paipa, C. A. (2021). Evolución del comercio electrónico: una perspectiva desde China y América Latina. Revista Brújula de Investigación, 9(17). 7-20. https://doi.org/10.21830/23460628.85 


\section{Introducción}

El dinamismo del mercado y las relaciones comerciales han llevado a la aparición del comercio electrónico, lo que ha fortalecido el crecimiento de los mercados internacionales que, ligado a los avances tecnológicos de los últimos años, ha transformado las relaciones comerciales e introducido nuevos conceptos como el B2B (Business to Business), el B2C (Business to Consumer), el C2C (Consumer to Consumer) y el B2G (Business to Government).

De esta manera, este tipo de innovaciones marcan la ruta para la especialización en nuevas técnicas de comercialización, las cuales son aprovechadas por los países que en su desarrollo incluyen tecnología con un enfoque expansionista, lo que permite generar mayor productividad y competitividad entre las empresas que buscan llegar ampliar su cobertura a nivel mundial, por medio de la reducción de sus costos por transacción con el objetivo de generar mayor rentabilidad.

Hay que mencionar, además, que de la mano con lo anterior ha surgido la economía digital que, desde 1990, forma parte de la sociedad de consumo, esto, por medio de plataformas especializadas y una amplia gama de aparatos tecnológicos, lo que ha permitido el desarrollo de otros medios de realización del comercio electrónico, como los servicios de pago en línea, publicidad, computación en la nube (cloud computing) y programas de facturación, que permiten transformar procesos en pro de la eficiencia de los mercados.

En el caso de China, el auge del comercio electrónico se dio en 1999, con el crecimiento de Alibaba Group y su plataforma Alibaba. com, inicialmente dentro del mismo país, atrayendo marcas locales en busca de crecimiento en mercados internacionales, es por esto que la expansión de internet ha propiciado también el desarrollo del comercio electrónico, con la particularidad de que en China el crecimiento ha sido exponencial.

Actualmente, el 69\% de los usuarios chinos realiza compras on-line, es decir, 533 millones de consumidores, lo que sitúa al país asiático como primer mercado mundial de comercio electrónico, con ventas anuales de 672.000 millones de dólares, más que el segundo y tercer clasificados juntos: Estados Unidos y el Reino Unido. (Tejero, 2018a)

Por otra parte, Latinoamérica en los últimos años ha incursionado en este nuevo modelo, de tal forma que entre 2013 y 2018 el comercio electrónico en Brasil, Argentina y México tuvo un crecimiento de más de un $100 \%$, pues son los países de la región con mayor infraestructura tecnológica, junto con Colombia. Asimismo, los países latinoamericanos aprovechan las oportunidades que brinda la continua actualización del mercado de consumo chino, diversifican sus exportaciones al país asiático y elevan la calidad y los precios de los productos exportados, indicó a Xinhua Sun Yanfeng, subdirector del Instituto de Estudios Latinoamericanos, dependiente del Instituto de Relaciones Internacionales Contemporáneas de China (Spanish. xinhuanet.com, 2019).

China es uno de los principales socios comerciales de la región Latinoamericana, teniendo en cuenta que el volumen del comercio bilateral alcanzó los 307.400 millones de dólares en 2018, con un aumento del 18,9\% respecto al año anterior, según los datos publicados por la Administración General de Aduanas (AGA) de China (Spanish.xinhuanet.com, 2019).

Ahora bien, el comercio electrónico proveniente de China seguirá evolucionando a mediano plazo y la presencia en la región Latinoamericana seguirá creciendo, dadas las condiciones y oportunidades actuales de la sociedad, 
permitiendo que se generen nuevas ideas de emprendimiento que lleven consigo un modelo de negocio basado en la economía digital.

Teniendo en cuenta lo expuesto anteriormente, surge las siguientes preguntas: ¿De qué manera el comercio electrónico ha transformado los modelos económicos de China y América Latina? ¿De qué manera China y América Latina se han adaptado al comercio electrónico.

En este orden de ideas, el presente escrito tiene como fin analizar el proceso evolutivo del comercio electrónico tanto en China como en América Latina; identificar los cambios que se han presentado en las relaciones comerciales; y determinar los factores que inciden en la adopción del comercio electrónico.

En el artículo se expondrán algunas teorías del comercio concernientes al desarrollo de nuevas tecnologías, luego se presentará un análisis de cada una de las regiones que intervienen en este proceso, mostrando de qué manera las estrategias planteadas por cada una han contribuido al desarrollo del comercio electrónico dentro de sus estructuras económicas.

Por último, se presentará, mediante cifras e indicadores, el alcance que ha tenido el comercio electrónico dentro de las economías latinoamericanas, con el fin de determinar si el comercio electrónico es viable para estas.

\section{Marco teórico}

\begin{tabular}{|c|c|}
\hline Teoría & Análisis aplicado a la investigación \\
\hline Mercantilismo & $\begin{array}{l}\text { Para iniciar se debe entender a lo que el mercantilismo se refiere, este sistema } \\
\text { económico, que se sostuvo hasta finales del siglo XVII, se caracterizó princi- } \\
\text { palmente por la intervención del Estado y el alto proteccionismo como política } \\
\text { comercial, lo que implicaba la implementación de una serie de medidas de unifi- } \\
\text { cación del mercado interno que tenían como finalidad la conformación de Esta- } \\
\text { dos-nación lo más fuerte posibles (Parra, 2017). }\end{array}$ \\
\hline $\begin{array}{l}\text { Ventaja } \\
\text { comparativa }\end{array}$ & $\begin{array}{l}\text { David Ricardo, en la teoría de la ventaja comparativa desarrollada a finales del } \\
\text { siglo XVII y principios del XIX, establece que los países deben especializarse } \\
\text { para el desarrollo de su producción, con el fin de aprovechar los bajos costos de } \\
\text { trabajo y demostrar que el comercio es mutuamente beneficioso para los actores } \\
\text { inmiscuidos en las nuevas tendencias de comercio global. Ricardo basa su teoría } \\
\text { en que la regla que rige el valor relativo de los bienes al interior de un país no es } \\
\text { la misma que regula el valor relativo de los productos que se intercambian entre } \\
\text { dos o más países (Rice, 2013). }\end{array}$ \\
\hline $\begin{array}{l}\text { Ventaja de los } \\
\text { factores }\end{array}$ & $\begin{array}{l}\text { Hecksher y Ohlin plantean la teoría de la ventaja de los factores, la cual estudia } \\
\text { la especialización de los países en el mercado internacional de acuerdo con la } \\
\text { dotación o disponibilidad de ciertos factores, bajo el supuesto de que en el pro- } \\
\text { ceso de producción y la combinación de tales factores cada país tiene los suyos } \\
\text { propios, es por ello que algunos países tienen el capital y otros la mano de obra. } \\
\text { En el caso chino la comercialización de productos a nivel mundial se da por la } \\
\text { abundancia en trabajo, permitiendo reducir los costes medios de los productos } \\
\text { (Legiscomex.com, s. f.). }\end{array}$ \\
\hline
\end{tabular}


Teoría

Nuevo comercio internacional

\section{Comercio} electrónico
La inserción de productos chinos en algunas economías latinoamericanas ha posibilitado el análisis de los patrones de comercio, la localización de la actividad económica y la competencia monopolística, esta última se da de manera imperfecta indicando que cada empresa puede tener factores diferenciadores tales como calidad, precio o valor agregado en los productos que ofrecen en el mercado. Esto hace que los países se vean obligados a adoptar nuevas técnicas de producción, con el fin de asimilar la tecnología y los fenómenos macroeconómicos que interfieren en la valoración de los capitales financieros, representados en instrumentos y herramientas que contribuyen a compensar los flujos de caja y a hacer realmente competitivas las economías internacionales, incluso las de países con reducidos factores de producción, según la connotación tradicional, pero con potenciales elementos innovadores que encajan dentro de la clasificación de los factores a la luz de la posmodernidad económica, ampliando el espectro de la conceptualización de la teoría económica en lo relativo al comercio internacional (Mayorga \& Martínez, 2008).

Estas nuevas tendencias de comercio, sumadas a la adopción de nuevas tecnologías, brindan una perspectiva diferente sobre la manera de llevar a cabo relaciones comerciales, adicionando un cambio significativo a la aplicación de los negocios tradicionales y haciendo de este una rama importante de la tecnología, donde se desarrolla una economía digital, tanto a nivel micro como macro, denominada comercio electrónico, el que, según David Van Hoose, puede ser definido como "cualquier proceso que conlleve intercambio de propiedad o el uso de derechos para bienes y servicios vía electrónica vinculando dispositivos y comunicando interactivamente dentro de la red” (Oropeza, 2018).

Este tema se aborda a inicios del siglo XXI, para poder llegar a desarrollar este tipo de comercio se debe tener en cuenta cómo se adquiere y se transmite la idea de este tipo de economía y su influencia en la producción y la determinación de los precios (Young, s. f.). En este sentido, Hayek señala que en este tipo de comercio

los individuos con racionalidad limitada pero con su información local de tiempo
y lugar deben asignar los recursos escasos entre una diversidad de objetivos; estas
acciones y elecciones a su vez informan a otros actores de forma tal que el mercado
se adapta efectivamente a los estímulos y responde al nuevo conocimiento sin que
ninguna persona tenga, o sea capaz de tener, todo el conocimiento requerido que lo
dirija a hacer eso.

Douglas North afirma que las instituciones son importantes en la regulación de

La economía institucional las relaciones de intercambio comercial, manteniendo la estabilidad de la economía, los medios de producción y la obtención de materias primas; de esta manera, el autor propone que deben existir relaciones entre las instituciones, las organizaciones tanto públicas como privadas y los empresarios, para poder tener una economía estable tanto interna como externamente (Urbano, Díaz \& Hernández, 2007). 


\section{Métodos}

El presente artículo de investigación fue desarrollado mediante una investigación mixta, en donde se mezclan los estudios cualitativos y cuantitativos con el fin dar respuesta a la pregunta principal desde el análisis de los datos obtenidos por medio de indicadores y cifras, lo que permite a la investigación brindar una perspectiva más cercana a la realidad, dicho método de investigación se define en la literatura de la siguiente manera:

Los métodos mixtos representan un conjunto de procesos sistemáticos, empíricos y críticos de investigación e implican la recolección y el análisis de datos cuantitativos y cualitativos, así como su integración y discusión conjunta, para realizar inferencias producto de toda la información recabada (metainferencias) y lograr un mayor entendimiento del fenómeno bajo estudio. (Hernández-Sampieri \& Mendoza, 2008).

\section{Resultados}

\section{Evolución del comercio electrónico como estrategia expansiva: China}

\section{EI nuevo paradigma del comercio en línea en China}

China, a través del tiempo, se ha adaptado a los cambios que la globalización ha impuesto en las maneras de hacer comercio, a diferencia de otros países desarrollados este ha optado por utilizar el comercio en línea como una estrategia para llegar a los mercados mundiales. De acuerdo con Krugman, China se adapta y toma ventaja de las necesidades de los mercados para desarrollar su oferta, así mismo, afirma que cuentan con la especialización de la mano de obra necesaria para ser competitivos.

Aunque las grandes compañías dominantes de este tipo de comercio son Amazon, Google y Facebook, entre otros, China desarro- lló sus propias empresas y redes sociales para el comercio, las cuales acaparan su mercado local desde 1997, cuando había 620.000 usuarios de internet (el 0,05\% de la población). Hoy son 802 millones, más que Estados Unidos y la India juntos, para luego llegar al comercio electrónico transfronterizo. El Gobierno Chino ha tenido mucho que ver con este nuevo modelo económico, ya que en 2013 decidió cambiarlo basándose en la inversión y las exportaciones, entre 2015 y 2016 China estableció 13 zonas piloto para simplificar los trámites aduaneros y logísticos relacionados con este tipo de operaciones, lo que trajo como resultados en los últimos cinco años: 1) un aumento en el PIB per cápita a una tasa interanual del 7,1\% (ppp); 2) más de 68 millones de habitantes en zonas rurales han salido de la pobreza; y 3) la clase media crece incesantemente (se espera que alcance los 550 millones de personas en 2022). En 2018, el comercio electrónico transfronterizo representó un 45,5\% del total de transacciones en línea, frente al 54,5\% del comercio electrónico doméstico. Pero se prevé que dichas cuotas sean del 50,7\% y el 49,3\%, respectivamente, en 2020 (Tejero, ICEX, 2018).

El comercio electrónico chino busca impulsar la producción interna y el desarrollo de las provincias dentro de algunas de sus regiones (Guangdong, Zhejiang, Pekín, Shanghái y Jiangsu), con el fin de que las exportaciones sean el principal objeto de crecimiento económico, haciendo que todas aquellas economías emergentes que basan su desarrollo en la producción y venta se desestabilicen, dado el principio de la ventaja competitiva (postulado por David Ricardo) donde no todos los países han focalizado su especialización, bien sea por capital o por mano de obra, por lo tanto China aumenta sus posibilidades de expansión en la economía digital. 
De este modo se inicia la ampliación del mercado local hacia el internacional, abriendo las puertas a procesos con grandes transformaciones, empezando desde la parte comercial, logística y de cadenas de valor; todo ello de acuerdo con la línea de pensamiento económico de Adam Smith y David Ricardo, es decir, considerando que la ampliación de mercados contribuye a perfeccionar los procedimientos de producción y puede llevar a la revolución industrial a un país cuyos recursos estaban sin desarrollar.

\section{Estrategias de expansión transfronteriza}

Para establecer estrategias, tanto de internacionalización como globales, se hace necesario considerar una serie de acciones que incentiven la participación en los mercados internacionales, aunque el éxito de esta empresa dependerá de las características y disponibilidad de recursos. Por lo tanto, es imprescindible identificar y establecer estrategias que permitan calificarlas como si realmente fuesen internacionales o globales, relacionando aspectos que determinen el nivel de participación o cuota de mercado que tengan, la ubicación de sus actividades y las acciones competitivas que realicen. La esencia de este proceso radica en la simultaneidad de tales acciones (Sarmiento, 2014).

En la actualidad, China ha incrementado su liderazgo desde varios sectores a través de los cuales desarrolla su mercado de una manera acelerada, con respecto a otros países que están en la carrera hacia la competitividad. Según Porter, la ventaja competitiva busca propiciar diferenciación entre los competidores, no obstante, el ciclo económico se basa en la variación de factores reales convergiendo en medio de una falta de proporcionalidad entre los distintos sectores productivos, dando como resultado el exceso de producción.
Por otra parte, el Gobierno chino muestra en su plan de desarrollo un proyecto llamado “Made in China 2025”, que busca alcanzar un modelo global de innovación a través de la tecnología de punta. Para el presidente chino, Xi Jinping, la tecnología será el nuevo campo económico de batalla y ha anunciado la transformación del modelo económico chino basado en exportaciones (Santos, 2018), esto se enlaza con los objetivos de Alibaba, el gigante asiático del comercio electrónico en el mundo que, desde 2016, desarrolla "Alibaba everywhere" con el que pretende potenciar el comercio electrónico tanto de manera local como en todo el mundo.

De igual manera, el desarrollo del comercio electrónico para las empresas chinas trae beneficios que incentivan la apertura de fronteras globales que fomentan el comercio internacional, en este sentido, uno de sus objetivos es mejorar la competencia entre los diferentes actores del mercado, en defensa del libre cambio y como incentivo para que surjan ventajas directas e indirectas, en concordancia con el pensamiento de John Stuart Mill. Por ello, el Consejo de Estado chino argumenta que es necesario

apoyar el desarrollo del comercio electrónico [en el país] mediante el fomento y la atracción de la participación de la inversión extranjera, todo ello con el fin de activar "la competencia del mercado", así que, por una parte, generalizar su uso, por otra parte activar y promover el sector de las ventas online internas (de ahí activar la competencia) para, posteriormente, conseguir que las empresas locales accedan a exportar también a partir de las ventas por internet a los consumidores globales. (Foro de Economia Digital, 2016, s. p.)

\section{A lo anterior habría se suma que}

China apuesta por el e-Commerce transfronterizo para promocionar el mercado interior de las ventas por internet, para ello, relaja su norma de no per- 


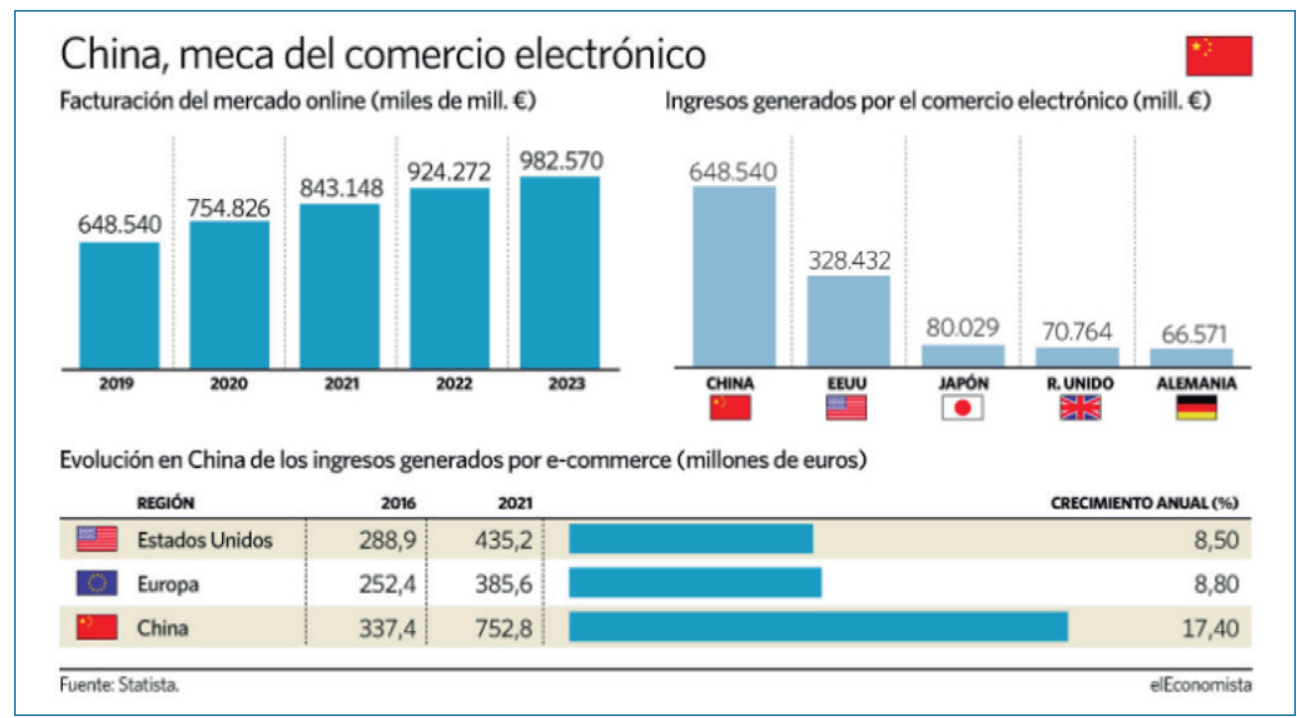

Figura 1. Comparativo entre China y Estados Unidos en comercio electrónico Fuente: La Quinta P (2019).

mitir la entrada de empresas con capital cien por cien extranjero, de esta manera, las autoridades han optado por permitir “algunas compañías” que se dediquen al manejo de datos y al comercio electrónico con el fin de promover ambos sectores. (Observatorio E-commerce, 2016, s. p.)

En la actualidad China se encuentra en una transición hacia las nuevas reglas del comercio fronterizo con el fin de que sus relaciones comerciales se realicen bajo parámetros claros y regulados internacionalmente.

Entrada a la modernización de las relaciones comerciales: Latinoamérica

El comercio electrónico en Latinoamérica ha crecido en los últimos años ofreciendo grandes posibilidades de acceso a los mercados internacionales, esto ha dado redundado en la reducción de costos que podrían encarecen los productos en un proceso de compra normal, ya sea para las empresas o para los consumidores finales, de este modo se abre una nueva forma de establecer relaciones comerciales internacio- nales. En este mismo contexto, desde 2013 Brasil ha sido uno de los países que se ha adaptado a este nuevo modelo, abarcando un $40 \%$ de las ventas totales realizadas por la región en este año, seguido por Argentina y México.

Por consiguiente, las relaciones comerciales que permite el comercio electrónico se han ido afianzando cada vez más dentro de la sociedad, de tal manera que este nuevo modelo permite dar una apertura al crecimiento de las economías que están en busca del desarrollo. Este proceso cumple, al menos, con dos puntos de la teoría planteada por Rostow, la cual postula cinco etapas de crecimiento que incluyen una transformación de la sociedad tradicional y la del consumo masivo.

De esta manera, se debe tener en cuenta la cantidad de usuarios que navegan por internet y pueden acceder a los servicios y compras en línea, con el fin de establecer el nivel de adaptación a las nuevas tecnologías por parte de los consumidores. 


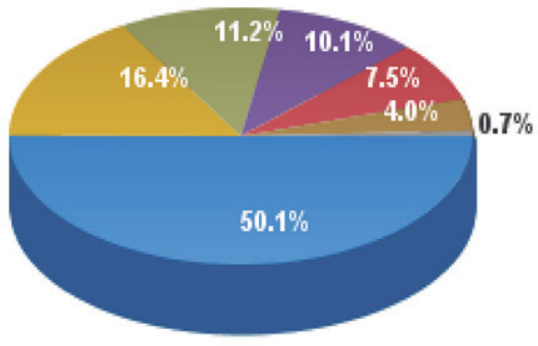

Asia $\mathbf{5 0 . 1} \%$

Europe $16.4 \%$

Africa. $11.2 \%$

Lat Am / Carib. 10.1\%

North America $\mathbf{7 . 5 \%}$

Middle East $\mathbf{4 . 0} \%$

Oceania / Australia $\mathbf{0 . 7} \%$

Figura 2. Usuarios de internet en el mundo por regiones Fuente: Spain Business School (2019).

\section{Potencial del mercado por comercio electrónico B2C}

Cada vez más las empresas están llegando directamente al cliente final, es por esto que el B2C se ha convertido en la manera más efectiva de llevar a cabo transacciones sin intermediarios, con varias ventajas para las partes, por un lado, la empresa vendedora puede obtener mayor utilidad, debido a que no existen costos por intermediarios, y el consumidor final paga un precio por debajo de lo normal con respecto al del mercado presencial, lo que también favorece a las empresas, pues logran un retorno de la inversión a corto plazo e invierten menos en infraestructura, priorizando el objetivo de la expansión del mercado ya sea a nivel local o internacional.

De esta manera, algunas de las cifras para Latinoamérica en este tipo de mercado han alcanzado cifras de hasta

US\$ 47.000 millones en 2015, un 24\% de incremento con respecto al año anterior. Además, América Latina cuenta con el mayor porcentaje de consumidores electrónicos (15\%) que realizan compras en línea exclusivamente en el extranjero comparado con Asia cuya cifra es de sólo 4\%. Brasil, México y Argentina suman $70 \%$ del valor de las transacciones en línea de la región. Brasil lidera el mercado con US\$ 15.000 millones en ventas en 2015, seguido por México y Argentina, con ventas estimadas en US\$ 13.000 millones y US\$ 5.000 millones, respectivamente. (Michalczewsky, 2019)

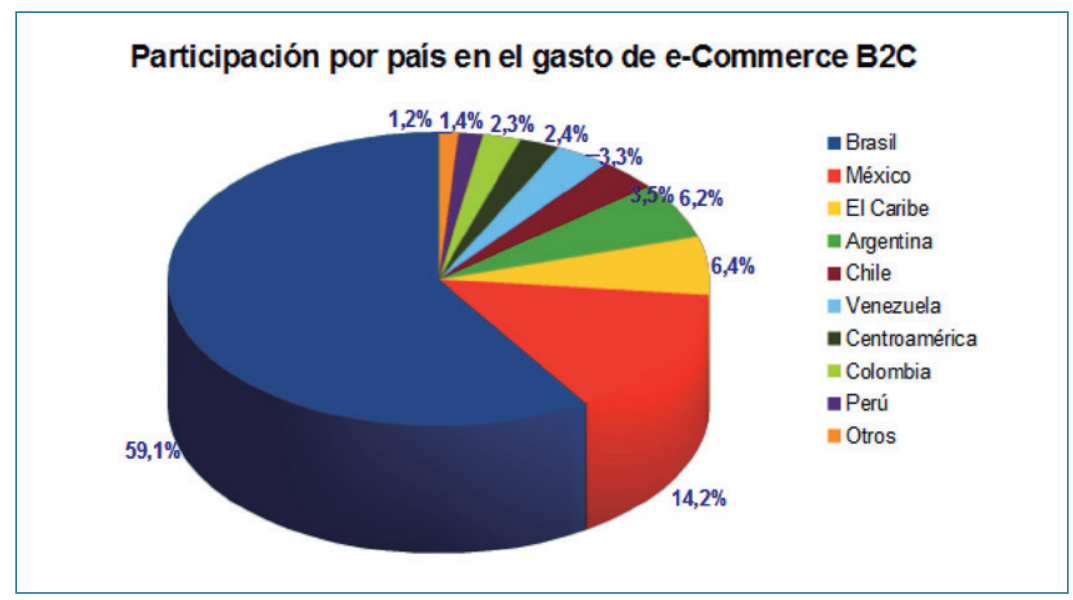

Figura 3. Participación por país en el gasto de comercio electrónico B2C

Fuente: BrainSINS (s. f.). 


\section{El comercio electrónico la nueva fuente económica de Latinoamérica}

El flujo de los mercados, se unifica para hacer que la economía crezca a nivel mundial, para el caso latinoamericano el comercio electrónico en los últimos años ha tomado fuerza, dando como resultado un crecimiento del $49 \%$ de los usuarios digitales que compran en línea, propiciando oportunidades para los países que han querido aprovechar las nuevas fuentes de compra y venta, basados en su producción, encontrando el rumbo hacia la especialización por medio del comercio electrónico.

\section{Como señala Mendoza}

las ventas de comercio electrónico en América Latina registraron un valor de aproximadamente 70.000 millones de dólares estadounidenses en 2019. Se estimó que para 2023 esta cifra podría ascender a unos 116.200 millones de dólares, lo que implicaría un incremento de casi 66 por ciento en relación a 2019. (2020)
Todo lo anterior evidencia la apropiación de las nuevas formas de hacer comercio con otros países, en pro de lograr un crecimiento económico que permita establecer nuevas formas de competitividad, no solo de manera local sino internacional, dando una alta expectativa a las empresas que se acogen a este modelo de comercio, con el fin de incrementar sus utilidades y reconocimiento en otros mercados.

A continuación, se presentan los resultados de una encuesta anual realizada por el Banco Interamericano de Desarrollo (BID), su Instituto para la Integración de América Latina y el Caribe (Intal) y Latinobarómetro, la cual tiene como propósito analizar las necesidades y demandas de Latinoamérica con respecto al potencial que tiene el comercio electrónico en la región. Según los datos de la encuesta

solo el 26\% de los latinoamericanos realiza compras por la web o está dispuesto a hacerlo. De estos, los que más compran y venden por la web son los hom-

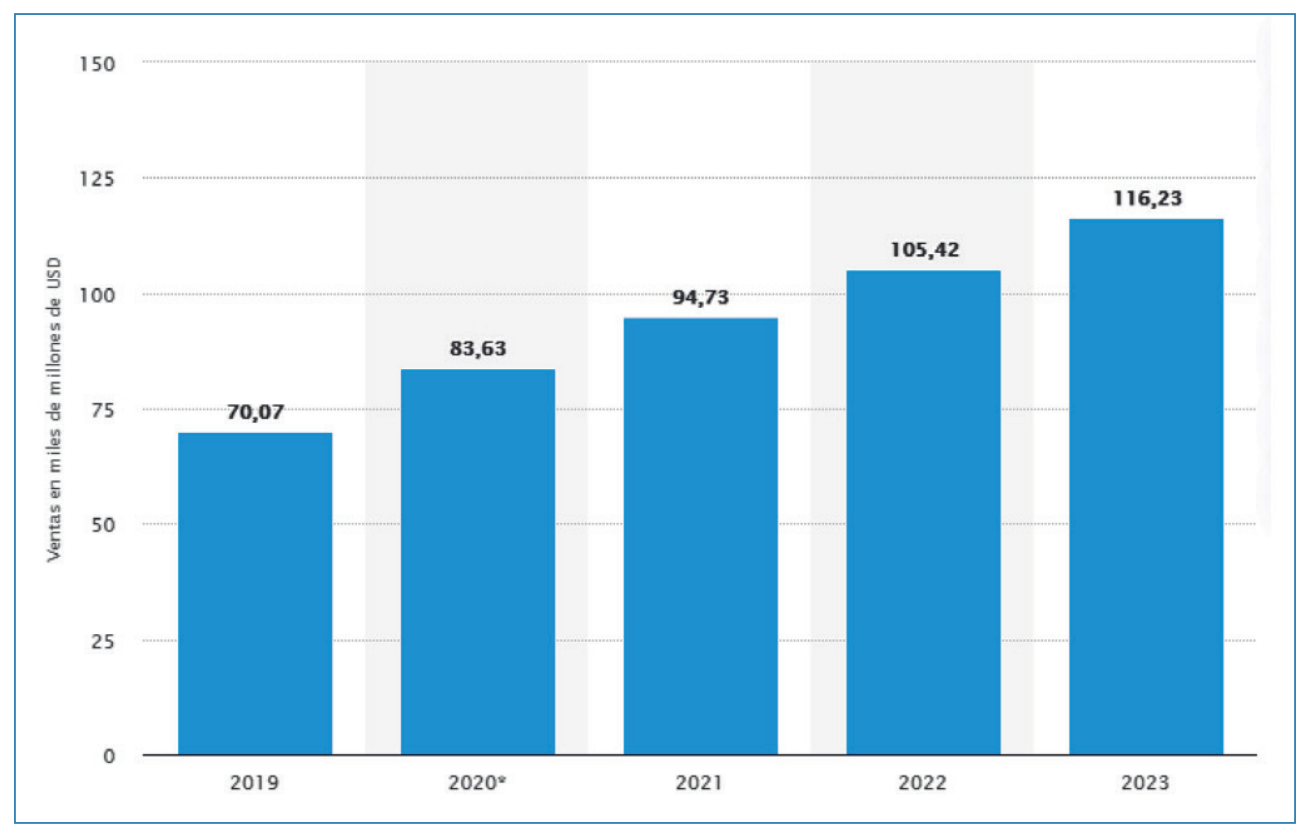

Figura 4. América Latina: ventas del comercio electrónico minorista 2019-2023

Fuente: Mendoza (2020). 
bres, los usuarios de smartphones y redes sociales, los de mejor situación económica y los más jóvenes. Los hombres superan a las mujeres (28\% vs $23 \%$ ) en su uso. Asimismo, los usuarios de smartphones y redes sociales superan (34\% y 31\% respectivamente) a quienes no utilizan estas tecnologías (26\% en promedio).

Entre los jóvenes menores de 25 años, el comercio electrónico llega al 31\%, y en los mayores de 65 años desciende al 14\%. Alcanza su máximo entre las personas de alta capacidad económica (33\%) y su mínimo entre las más desfavorecidas (13\%).

Los países con población más ávida por utilizar el comercio electrónico son Venezuela y Costa Rica (47\% y 40\% respectivamente), mientras que los menos dispuestos a su utilización son Ecuador, Nicaragua y El Salvador (entre 13\% y 15\%).

El comercio electrónico goza de más aceptación en los países de más alto PBI per cápita, mayor cantidad de población usuaria de internet, y mejor índice de desarrollo humano, como Argentina, Chile, Uruguay, Costa Rica y Colombia.

El uso de plataformas digitales para generar ingresos es aún incipiente. Solo el 9\% de los latinoamericanos reconoce haber hecho uso de ellas. El mayor nivel de utilización se presenta en Costa Rica (22\%) y República Dominicana (20\%), que contrasta con mínimos de 2\% de utilización en Nicaragua o 3\% en Paraguay.

Seis de cada 10 latinoamericanos interpretan que el uso de la información privada en internet con fines comerciales representa una violación de un derecho humano básico. La cuestión de la privacidad y el uso de la información preocupa más a las personas de mayor nivel socioeconómico (68\% entre los grupos más favorecidos) y a los usuarios de smartphones (69\%) y redes sociales (67\%).

Adicionalmente, la encuesta encontró que el 73\% es usuario de redes sociales, el 89\% tiene teléfono móvil, y el 47\% tiene Smartphone.

Los teléfonos celulares se han vuelto tan extendidos como el agua potable y los Smartphone están más presentes que algunas tecnologías mucho más antiguas como los automóviles, el agua caliente o la calefacción. De hecho, el 80\% de los latinoamericanos que solo tienen una comida por día a menudo posee un teléfono celular y el 32\% posee un Smartphone. La tecnología digital se ha convertido en una forma de conectar hasta los más pobres. (Forbes Staff, 2020)
Actualmente, la tecnología supone adelantos y desarrollo productivo, logrando que las empresas sean más eficientes, con el fin de que los costos sean bajos, para que la efectividad sea más significativa, desarrollando de manera indirecta nuevas plazas laborales como el marketing, que junto con la organización empresarial complementan y generan valor agregado a los productos o la forma en que llegan a los consumidores. Según la publicación del portal de empleo "elelmpleo.com"

bajo el título "Comercio electrónico, una nueva plaza para emplearse": "Encabezando la lista de las nuevas oportunidades laborales del comercio electrónico, está el 'e-commerce manager', máximo responsable de la plataforma de venta en línea, que define, realiza seguimiento y ejecuta las estrategias de venta 'online' y puede estar ganando entre $\$ 3.50$ 0.000 a \$20.000.000 según su experticia”, comenta Andrés Díaz Granados, executive manager de DNA Human Capital. (Castro, 2019)

\section{El comercio electrónico en la coyuntura actual}

En medio de los pronósticos acerca de la futura crisis económica en el mundo, Latinoamérica ha sabido potencializar su mercado, según el sitio web del diario La República

el comercio electrónico atraviesa su mejor momento en América Latina con crecimientos superiores a 300\%. Según un estudio de Kantar a nivel regional, en la primera semana de confinamiento, la penetración del comercio electrónico registró un aumento de $100 \%$, mientras que en la cuarta semana el alza fue de $387 \%$. (Rubio, 2020)

Por lo anterior, se evidencia que los consumidores optaron por el comercio electrónico dadas las condiciones en las cuales se está desarrollando la pandemia, un caso puntual de esta región es Colombia, pues es parte de la transformación que se vive actualmente y que ha logrado consolidarse en este tipo de comercio. 
COMPORTAMIENTO DEL COMERCIO ELÉCTRÓNICO EN LA COYUNTURA ACTUAL
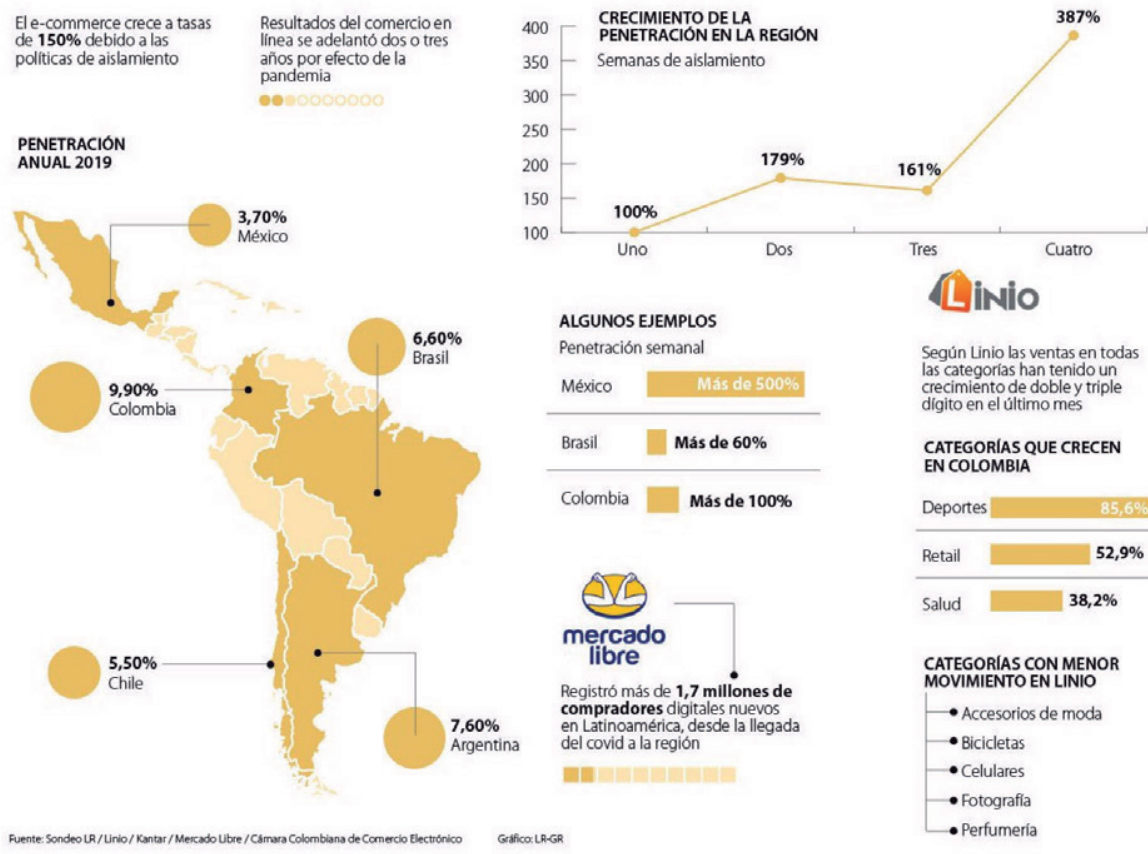

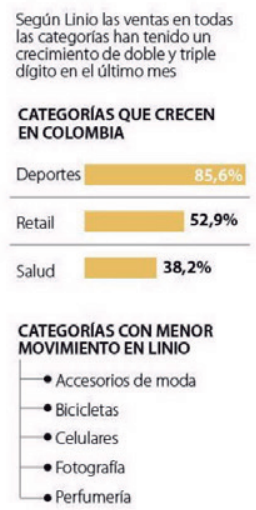

Según Linio las ventas en todas las categorias han tenido un crecimiento de doble y triple
dign el último mes EN COLOMBIA

CATEGORIAS CON MENOR MOVIMIENTO EN LINIO

- Accesorios de moda

- Perfumería

Figura 5. Comportamiento del comercio electrónico en la coyuntura actual Fuente: Rubio (2020).

Como señala Forero, "con el avance de la transformación digital en los e-commerce, las empresas y startups del país se han dado cuenta de la importancia de adoptar una estrategia de ventas online para atender a una población cada vez más digital” (2020).

Por otra parte, China y Colombia firmaron un memorando de entendimiento con el fin de promover la cooperación en comercio electrónico, según el ministro de Comercio, Industria y Turismo, José Manuel Restrepo, “China es el segundo socio comercial de Colombia y aún tenemos mucho espacio para crecer en exportaciones nacionales hacia China. En ese escenario, las plataformas de comercio electrónico son instrumentos que nos ayudarían a cumplir este propósito" (La Opinión, 2019), de esta manera Colombia se direcciona hacia la economía digital.
Por otra parte, la Cámara Colombiana de Comercio Electrónico y el Ministerio de Tecnologías de la Información y las Comunicaciones (MinTIC) dieron a conocer el segundo informe sobre el comportamiento del comercio electrónico durante las medidas de cuarentena, en el que se destaca un importante crecimiento de la actividad durante las semanas comprendidas entre el 5 de abril y el 9 de mayo.

El estudio demuestra que el comercio electrónico se viene reactivando, llegando a índices similares a los que se tenía antes del inicio de la pandemia en el país. En la semana del 3 a 9 de mayo pasados, se registraron 4,17 millones de transacciones en línea, que corresponden a $\$ 495$ mil millones. Cifra cercana a los niveles presentados en los últimos días de febrero, antes del inicio de la emergencia sanitaria, cuando se realizaron 4,22 millones de transacciones digitales, que corresponden a $\$ 535$ mil millones en ventas.

El crecimiento del comercio electrónico es del 73\% entre el 5 de abril y el 3 de mayo, en comparación con la última semana de marzo, se explica debido 
a la robustez presentada por las cadenas de abastecimiento de estos bienes y a la claridad en la operación que se dio por parte del Gobierno Nacional y los gobiernos locales, que permitieron el incremento del consumo de bienes de primera necesidad y consumo ordinario. (Semana, 2020)

De esta manera, cabe resaltar que Latinoamérica está inmersa en la economía digital, la cual promueve el uso de las tecnologías de la información y la comunicación (TIC), y genera desarrollo e inclusión, promoviendo las nuevas ideas de emprendimiento con miras a la internacionalización, brindando conectividad con todas partes del mundo a todas las personas que tienen acceso a internet. Según Naciones Unidas (2013), la economía digital —medida por el gasto en TIC, el número de usuarios de internet y la diversificación de sus usos - ha adquirido una creciente importancia en los países de América Latina, es más, durante la última década, la región se ha convertido en un mercado

emergente en el uso de aplicaciones TIC por las empresas, el gobierno y los individuos, y el gasto en este rubro alcanzó a 295000 millones de dólares en 2011, cerca de 5,2\% del PIB (Gartner, 2012). El gasto de América Latina fue equivalente a $8 \%$ del total mundial, como resultado de un crecimiento sostenido que alcanzó a 12,1\% en 2011, el segundo más alto después de la región de Asia Pacífico. El gasto mundial en TIC se descompone en telecomunicaciones (47\%), servicios TIC (24\%), dispositivos (17\%), software (8\%) y centros de datos (4\%). (CEPAL, 2013)

\section{Discusión}

El comercio internacional trae consigo diferentes perspectivas de desarrollo para las regiones estudiadas en la investigación, cada una de ellas ha logrado adaptarse a este modelo de acuerdo a las condiciones, culturales, económicas, sociales y políticas que provee cada uno de sus sistemas, sin embargo, esta investigación evidenció la gran brecha que existe entre las dos regiones en cuanto a las estrategias utilizadas para lograr que el cambio sea positivo en sus economías. Como se planteó en el marco teórico, puede decirse que China aprovecha la ventaja productiva para poder llegar al mercado mundial y de esta manera liderar el mercado, por medio del comercio electrónico y sus nuevas relaciones comerciales.

Por otro lado, Latinoamérica se encuentra promoviendo este tipo de comercio pese a la poca aceptación de sus habitantes, debido al choque cultural y el miedo al fraude - que es percibido casi como una característica de este tipo de comercio- - por lo que se puede decir que esta región está en una primera fase de adaptación. La coyuntura debida a la pandemia ha permitido que el comercio electrónico se lleve a efecto de una manera más acelerada, con el fin de mantener la economía estable.

\section{Conclusiones}

El comercio electrónico en China y América Latina hace que sus economías logren adaptarse tanto a las nuevas tecnologías como a la optimización de la producción, con el objeto de obtener una posibilidad de llegar al mercado mundial, por tal razón incentiva a las empresas a hacer parte de la economía digital y abre la puerta al mercado mundial, por medio de la venta de productos y servicios; además, incluye a los consumidores, ya que los motiva a participar de manera directa desde la comodidad de su hogares o sitios de trabajo.

Las relaciones comerciales han cambiado de tal manera que, a lo largo del desarrollo del comercio electrónico, se han incluido nuevos términos que inciden en la realización de los negocios, pues ya no se dan de persona a persona, sino por medio de plataformas, lo que ha 
llevado a que el B2C logre consolidarse, puesto que permite que se dé una relación directa entre las personas y las empresas, logrando así reducir notablemente los costos.

La adopción del comercio electrónico en China se da gracias a que es pionera en el desarrollo del mismo, esto hace que sus procesos de producción resulten eficientes para llegar al mercado internacional, ya que la tecnología va de la mano con en el desarrollo de las nuevas prácticas. En adición, culturalmente, el gigante asiático ha logrado acoplarse a los cambios que ha traído consigo la globalización y su enfoque trasciende la economía local. Por otra parte, América Latina se está adaptando a la transformación digital en lo que atañe a la compra y venta de productos y servicios, cabe resaltar que la cultura latinoamericana es más cerrada a los procesos de transformación económica, pues aún existen temores en la utilización de las nuevas tecnologías.

\section{Agradecimientos}

La autora agradece a la Fundación Universitaria Empresarial de la Cámara de Comercio de Bogotá por el apoyo brindado para la realización de este artículo.

\section{Declaración de divulgación}

La autora declara que no existe ningún potencial conflicto de interés relacionado con el artículo. Los puntos de vista y los resultados de este artículo pertenecen a la autora y no reflejan necesariamente los de las instituciones participantes.

\section{Financiamiento}

La autora no declara fuente de financiamiento para la realización de este artículo.

\section{Sobre la autora}

Camila Andrea Paipa Bolaños. Fundación Universitaria Empresarial de la Cámara de Comercio de Bogotá. Contacto: cpaipa@ uniempresarial.edu.co

\section{Referencias}

BrainSINS (s. f.). Y, ¿qué es del e-Commerce en Latinoamérica? https://www.brainsins.com/es/ blog/y-que-es-del-e-commerce-en-latinoamerica/102018

Castro, J. (5 de diciembre de 2019). elempleo. https:// www.elempleo.com/co/noticias/tendencias-laborales/comercio-electronico-una-nueva-plaza-para-emplearse-5979

CEPAL. (2013). Economia digital para el cambio estructural y la igualdad. Santiago de Chile: Naciones Unidas.

Forbes Staff. (9 de marzo de 2020). Centroamerica Forbes. https://forbescentroamerica. com/2020/03/09/termometro-del-comercio-electronico-en-america-latina/

Forero, T. (3 de febrero de 2020). rockcontent. https:// rockcontent.com/es/blog/comercio-electronico-en-colombia/

Foro de Economía Digital. (12 de febrero de 2016 ). Observatorio ecommerce. https://www.observatorioecommerce.com.co/china-abre-fronteras-para-fomentar-el-ecommerce/

Huerta, R. (17 de octubre de 2017). BID - Mejorando Vidas. https://blogs.iadb.org/integracion-comercio/es/comercio-electronico-america-latina/

ICEX. (20 de octubre de 2017). eMarket Services.

La Quinta P (2019). China arrasa en Internet y duplica ya el negocio de EEUU en comercio electrónico. https://laquintapicai.blogspot.com/2019/11/china-arrasa-en-internet-y-duplica-ya.html

La Opinión. (3 de agosto de 2019). La Opinión. https://www.laopinion.com.co/economia/colombia-y-china-acuerdan-apoyo-para-e-commerce181577\#OP

Legiscomex.com. (s. f.). Legiscomex. https://www. legiscomex.com/Documentos/abccomercio-dotacion-factores

Mayorga, J. \& Martínez, C. (2008). Paul Krugman y el nuevo Comercio Internacional. Criterio Libre, (8), 73-86. 
Mendoza, J. (25 de junio de 2020). América Latina: ventas del comercio electrónico minorista 20192023. https://es.statista.com/estadisticas/637442/ latinoamerica-ventas-de-comercio-electronico-minorista-de--a-2019/\#: :text=Las\%20 ventas\%20de\%20comercio\%20electr\%C3\%B3nico,ciento\%20en\%20relaci\%C3\%B3n\%20 a\%202019.

Michalczewsky, K. (2019). Banco Interamericado de Desarrollo. https://conexionintal.iadb. org/2018/05/30/ideas-4/

Naciones Unidas. (marzo de 2013). https://repositorio.cepal.org/bitstream/handle/11362/35408/1/ S2013186_es.pdf

Observatorio e-commerce (12 de 02 de 2016). https:// www.observatorioecommerce.com.co/china-abre-fronteras-para-fomentar-el-ecommerce

Oropeza, D. (2018). ¿ Qué es el comercio electrónico? En D. K. Oropeza, La competencia económica en el comercio electrónico y su protección en el sistema jurídico mexicano (pp. 1-4). https://archivos.juridicas.unam.mx/www/bjv/ libros/10/4667/4.pdf

Parra, C. (2017). Legiscomex. https://www.legiscomex.com/Documentos/abccomercio-mercantilismo

Reyes, G. (2009). Teorías de Desarrollo Económico y Social: Articulación con el Planteamiento de Desarrollo Humano. Tendencias, 10(1), 117-142.

Rice, E. (2013). El papel de la Ventaja Competitiva en el desarrollo económico de los países. Análisis económico, 28(69), 55-78.
Rubio, P. (4 de 05 de 2020). La República. https:// www.larepublica.co/globoeconomia/e-commerce-ha-crecido-mas-de-300-en-latinoamerica-en-medio-de-la-pandemia-3000424

Spain Business School (2019). https://blog.spainbs. com/2019/05/562/dia-de-internet-celebremos-la-transformacion-digital

Santos, C. (agosto de 2018). China: Ambiciosas estrategias de expansión y oportunidades para América Latina. Revista Percápita. https://revistapercapita.com/china-estrategias-expansion/

Sarmiento, S. (2014). Estrategias de internacionalización y globales para países en desarrollo y emergentes. Revista Dimensión Empresarial, 12(1), 111-138.

Semana. (6 de junio de 2020). Semana. https:// www.semana.com/nacion/articulo/coronavirus-en-un-73--crecio-el-comercio-electronico-durante-cuarentena/679062

Spanish.xinhuanet.com. (01 de febrero de 2019). Xinhua Español. http://spanish.xinhuanet.com/201902/01/c_137792833.htm

Tejero, C. (2018a). Comercio electrónico transfronterizo: China rompe los esquemas. Isex. es. https://www.icex.es/icex/es/Navegacion-zona-contacto/revista-el-exportador/mercados/ REP2018797484.html

Xue, X. (07 de marzo de 2016). spanish.people.cn. e http://spanish.peopledaily.com.cn/n3/2016/0307/ c31621-9026386.html

Young, G. (s. f.). Hayek y el comercio electronico: conocimiento y complejidad en el siglo XXI. http:// www.eseade.edu.ar/wp-content/uploads/2016/08/ Young.pdf 